\title{
Nanoscale Strainability of Graphene by Laser Shock-Induced Three- Dimensional Shaping
}

\author{
$\mathrm{Ji} \mathrm{Li}^{\dagger, \dagger}$ Ting-Fung Chung, ${ }^{\dagger, \|}$ Yong P. Chen, ${ }^{\dagger, \S, \|}$ and Gary J. Cheng* ${ }^{\dagger, \dagger}$ \\ ${ }^{\dagger}$ Birck Nanotechnology Center, ${ }^{\ddagger}$ School of Industrial Engineering, ${ }^{\S}$ School of Electrical and Computer Engineering, and ${ }^{\| D e p a r t m e n t}$ \\ of Physics, Purdue University, West Lafayette, Indiana 47907, United States
}

ABSTRACT: Graphene has many promising physical properties. It has been discovered that local strain in a graphene sheet can alter its conducting properties and transport gaps. It is of great importance to develop scalable strain engineering techniques to control the local strains in graphene and understand the limit of the strains. Here, we present a scalable manufacturing process to generate three-dimensional (3D) nanostructures and thus induce local strains in the graphene sheet. This process utilizes laser-induced shock pressure to generate $3 \mathrm{D}$ tunable straining in the graphene sheet. The size dependent straining limit of the graphene and the critical breaking pressure are both studied. It is found that the graphene film can be formed to a circular mold ( $\sim 50 \mathrm{~nm}$ in diameter) with an aspect ratio of 0.25 and strain of $12 \%$, and the critical breaking pressure is $1.77 \mathrm{GPa}$. These values were found to be decreasing with the increase of mold size. The local straining and breaking of graphene film are verified by Raman spectra. Large scale processing of the graphene sheet into nanoscale patterns is presented. The process could be scaled up to roll-to-roll process by changing laser

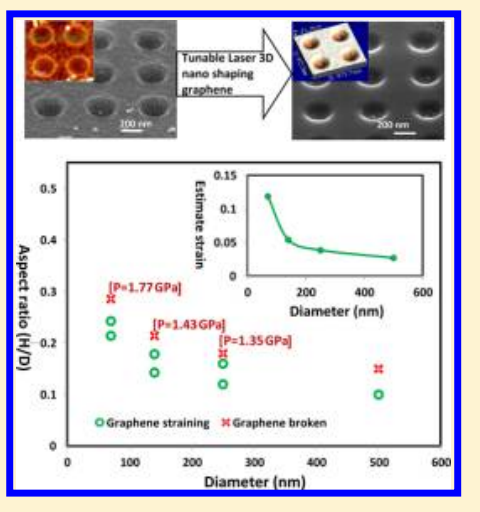
beam size and scanning speed. The presented laser shock straining approach is a fast, tunable, and low-cost technique to realize strain engineering of graphene for its applications in nanoelectrical devices.

KEYWORDS: Laser shock-induced straining, 3D nano shaping, graphene, strainability

$G$ raphene, an atom-thick layer of carbon, has attracted significant attention since its experimental discovery in 2004. ${ }^{1}$ As the mother of all graphitic materials such as fullerenes, carbon nanotubes, and graphite, graphene possesses unusual properties, including structural perfection, low density, excellent electrical properties, ${ }^{2,3}$ zero effective mass near the Dirac point, ${ }^{4}$ long mean free path, ${ }^{5}$ and superior mechanical properties. $^{6}$ However, unpatterned graphene has limited functionality ${ }^{4,7}$ because of its zero bandgap. One approach has been developed which patterns graphene into nanoribbons and dots. ${ }^{8,9}$ However, reliability and scalability remain problems for graphene nanopatterning. The high disorder level of the patterned graphene and sensitivity of transport to the edge profile, as well as the difficulty in controllable and repeatable fabrication of such microstructures with sharply defined edges, severely limit the application of graphene nanoribbons in electronics. Recently, more focus has been put on another approach, straining, to tailor the electronic properties of graphene. ${ }^{10-12}$ It has been shown that all of the characteristics of graphene ribbons and dots could be obtained by generation of local strain in graphene lattice, which allows one to make an all-graphene circuit. ${ }^{10}$ Meanwhile, certain configurations of strains in graphene are equivalent to a magnetic field, which could be very high. ${ }^{12,13}$ Thus, it is of great importance to develop scalable manufacturing techniques to precisely induce the amounts and types of strains in graphene films. It has been proposed that this can be achieved by suspending graphene on a substrate with different geometrical patterns, such as grooves, creases, steps, and wells. ${ }^{10}$ However, it is difficult to control the strain level in graphene that varies as a result of both transfer processes and operation errors.

Here, we present a scalable manufacturing process to precisely induce local strains in the graphene sheet. This process utilizes laser-induced shock pressure to generate threedimensional (3D) shaping in the graphene sheet. The graphene sheet is conformally pressed onto the mold patterns and takes the shape of the patterns, resulting tunable strains in the graphene sheet. The graphene is attached to the mold surface after the laser shock, due to the van der Waals force. As a result, the 3D strained shapes remain after the process. The strainability of the graphene film is studied by varying the forming depth in order to find out the maximum straining aspect ratio (depth over diameter in the deformed area) as a function of mold diameters. The critical pressure needed to break the graphene film as a function of size of holes is also studied. Raman spectroscopy is used to verify the local straining and breaking of the graphene film.

The laser shock-induced graphene straining process is illustrated in Figure 1. The nanopatterns on Si wafer were fabricated by focused ion beam (FIB) milling or e-beam lithography. Chemical vapor deposition (CVD) grown single layer graphene film is transferred on to the $\mathrm{Si}$ mold. Laser dynamic forming (LDF) process ${ }^{14,15}$ is then carried out on the graphene film, as seen in Figure 1a. When the laser pulse

Received: May 15, 2012

Revised: August 2, 2012

Published: August 9, 2012 


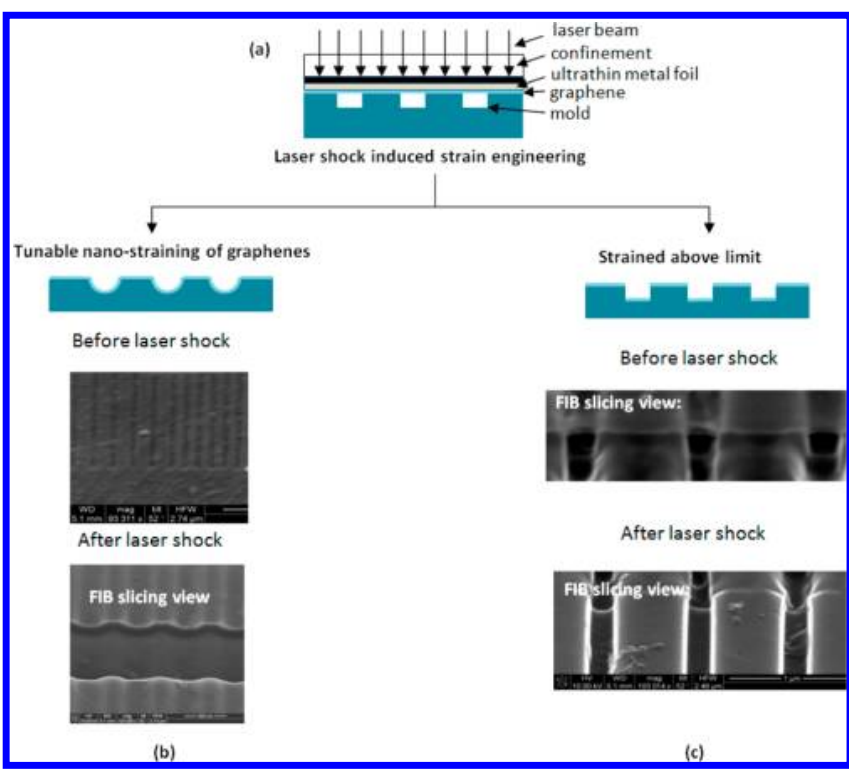

Figure 1. Illustration of the laser shock induced straining of graphene. (a) Setup of of the laser-induced strain engineering; (b) tunable 3D nanostraining of the graphene film when the strain limit is not exceeded. (c) Graphene straining above the strain limit.

transmits through the confinement and irradiates the ablative coating, it quickly vaporizes and ionizes into plasma. The expansion of the plasma is confined and bounced off by the confining media, generating a strong shock wave, which provides a strong momentum to shape the graphene film onto the mold surface. The thin aluminum foil is positioned between ablative coating and graphene film to prevent direct contact of ionized plasma on graphene and also effectively transfer the shock pressure onto the graphene sheet. It is been found that metals experience superplasticity during high strain rate forming, which allows the metal foils deforms into the nanoscale 3D mold. Therefore, after the impact of the laserablated shock pressure, the metal foil, and graphene sheet is pressed into the patterned on the mold and firmly attached to the substrate surface. The duration of the shock is only tens of nanoseconds, and the process involves one step operation. In order to apply uniform pressure on large areas, a beam diffuser could be used to flatten the Gaussian profile of fundamental mode laser beam. With a motion controlled table or a beam scanner and high pulse frequency, the laser shock-induced straining process could used to apply large scale shaping of graphenes at high speed.

Figure $1 \mathrm{~b}$ shows the results of laser shock induced shaping on graphene layer. The FIB slicing view clearly shows the graphene's profile before and after shaping. When the shock pressure is lower than a critical value, graphene sheet was elastically strained and conformally attached on the silicon mold by van der Waals force. When the shock pressure is high enough, the graphene sheet experiences breaking around the edges of the mold because its straining limit is exceeded, as shown in Figure 1c. The magnitudes of the strains are tunable based on the mold geometry. This study will investigate the critical straining and pressure levels that the graphene film can attain, beyond which breaking of the graphene will take place. The breaking of graphene film has other applications, such as patterning of graphene; ${ }^{16}$ however, this study is focused on the straining range of graphene and the shaping level that could be achieved.

More results of laser nanoshaping and straining of graphene are given in Figure 2. Examples of the original sample with graphene film lying over nano molds are illustrated in Figure 2a,d. Figure 2a shows a typical scanning electron microscopy (SEM) image of the sample on channel mold. The width of the

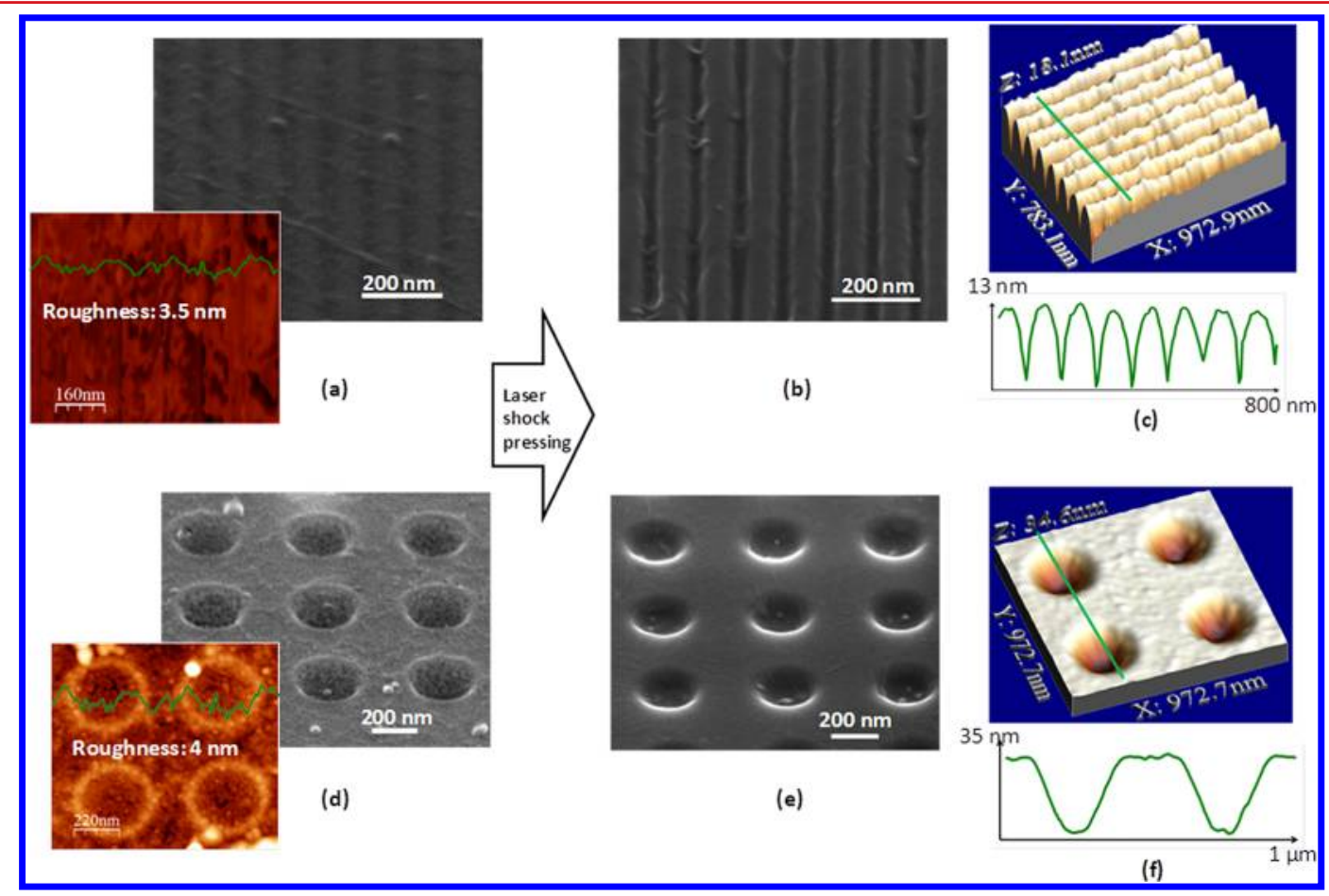

Figure 2. SEM and AFM images of (a) graphene film transferred to a silicon mold patterned with arrays of channels before laser shock shaping; (b,c) graphene film deformed into channels; (d) graphene film transferred to a silicon mold patterned with circular wells; (e,f) graphene film deformed into wells. The samples are tilted $52^{\circ}$ for SEM observation. 
mold is about $30 \mathrm{~nm}$ and the depth is about $12 \mathrm{~nm}$. Some poly(methyl methacrylate) (PMMA) residuals are observed on the surface of the graphene, which are left over from the PMMA-assisted transfer process of the CVD grown graphene film. Morphology of the suspending graphene film on Si mold is observed by atomic force microscopy (AFM). It can be seen that the surface of the graphene film is very flat with an overall roughness characterized as $3.5 \mathrm{~nm}$. Figure $2 \mathrm{~d}$ shows the SEM and AFM images of graphene film suspending on circular molds. Normally graphene film suspending on mold cavities tends to slightly bend into and thus stretch across the openings due to the van der Waals attraction of the membrane to the substrate. ${ }^{18}$ However, the stretching of the graphene film is very small in this study due to the small size of the mold and is hardly seen from the height profile information. Therefore, the stretching and prestressing in the graphene film can be neglected.

During the laser shock operation, the graphene film is pressed onto the nanomolds by the strong and short laser shock pressure, resulting in local straining of the film. The laser shock pressure value can be estimated by Fabbro's model ${ }^{19}$ in which the peak pressure value in confined laser ablation process is given by

$$
P(\mathrm{GPa})=0.01\left(\frac{\alpha}{2 \alpha+3}\right)^{1 / 2} Z^{1 / 2}\left(\frac{\mathrm{g}}{\mathrm{cm}^{2} \mathrm{~s}}\right) I_{0}^{1 / 2}\left(\frac{\mathrm{GW}}{\mathrm{cm}^{2}}\right)
$$

where $\alpha$ is the fraction of absorbed energy (typically equals to $0.1), I_{0}$ is laser intensity, and $Z$ is calculated by

$$
\frac{2}{Z}=\frac{1}{Z_{1}}+\frac{1}{Z_{2}}
$$

where $Z_{1}$ and $Z_{2}$ are the impedances of the confining media (glass) and the substrate material (aluminum foil), respectively.

As the confining media and the metallic foil are kept the same in the experiments, the laser shock pressure is controlled by adjusting the laser intensity. The laser intensity used in this study ranges from 0.2 to $0.7 \mathrm{GW} / \mathrm{cm}^{2}$, and the calculated laser shock pressure ranges from 1.0 to $1.8 \mathrm{GPa}$. The actual pressure applied on the graphene film is slightly smaller than the laser shock pressure due to the attenuation in the aluminum foil, which is neglected in this study. The laser intensity of 0.2 to 0.7 $\mathrm{GW} / \mathrm{cm}^{2}$ could be achieved on $3 \mathrm{~mm}$ area with pulse energy of 70 to $230 \mathrm{~mJ}$ and pulse duration of $5 \mathrm{~ns}$ for a Gaussian beam profile. Larger area can be processed using a beam scanner.

As shown in both SEM images (sample is tilted $52^{\circ}$ ) and AFM images in Figure 2b,c,e,f, after the laser shock shaping process, the graphene film is no longer suspending on the nanomolds (channels or holes), but is attaching to the curved surface of the Si substrate and nicely takes the shape of the molds. The laser intensity used is $0.45 \mathrm{GW} / \mathrm{cm}^{2}$, corresponding to an estimated pressure of $1.5 \mathrm{GPa}$. As the graphene film is fixed at the bar positions of the substrate, these parts of the film has little change before and after the laser shock operation. In the contrast, the freestanding parts of the film originally suspending on the mold cavities are suddenly hit and pressed by the pressure impact, resulting in local straining of the membrane. The local straining in the graphene layer can be controlled by varying prefabricated patterns on the substrate and appropriate pressure shock. The AFM characterization also gives the height profiles of the deformed graphenes. Examples are shown in Figure $2 c, f$, which give an average forming depth of $12 \mathrm{~nm}$ for the $30 \mathrm{~nm}$ wide channel mold, and a depth of 31 $\mathrm{nm}$ for the $250 \mathrm{~nm}$ diameter circular mold, and the graphene film is well shaped without fracture in these two cases.

During the deformation process, there is a critical straining level that the graphene film can attain, beyond which fracture of the film will take place. The critical forming depth is related to the mold shape and size. In order to study the critical shaping level and its relationship with the nanomold size, circular molds with different depth were used for the graphene shaping. The forming results were observed by SEM in the order of increasing forming depth, and the straining area and breaking area were identified, as given in Figure 3. Aspect ratio (forming depth over diameter) is used to represent the forming level of the graphene film. Figure $3 a$ shows the shaping results of graphene film into the circular molds with increasing aspect ratio from left to right. When the aspect ratio is low (0.1), the graphene film is well deformed. Then, fracture of the film takes

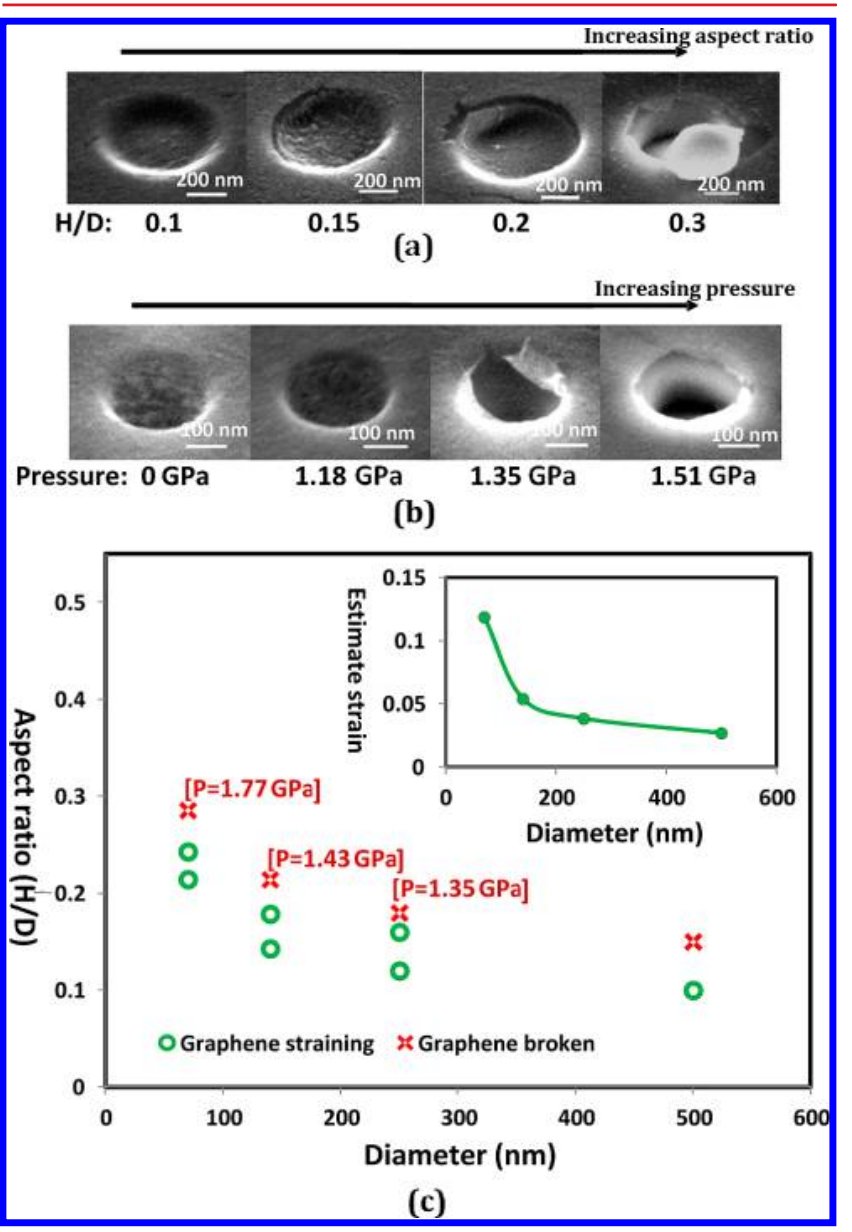

Figure 3. (a) SEM iamges (tilt $52^{\circ}$ ) of deformed graphene film in circular mold with increasing depth (diameter is kept at $500 \mathrm{~nm}$ ). The aspect ratio from left to right is $0.1,0.15,0.2$, and 0.3 , respectively. The scale bar is $200 \mathrm{~nm}$. (b) SEM images (tilt $52^{\circ}$ ) of graphene film after being pressed by increasing laser shock pressure. The mold diameter is $250 \mathrm{~nm}$, and the depth is much larger than the maximum forming depth that the graphene film can attain. The shock pressure applied from left to right is estimated as 0 (original), 1.18, 1.35, and 1.51 GPa, respectively. The scale bar is $100 \mathrm{~nm}$. (c) The relationship between circular mold diameter and the maximum aspect ratio that the graphene film can attain. The inlet is the relationship between the diameter and estimate strain of the safe points (green circle). The critical breaking pressure of graphene at each hole diameter is given as $P$. 


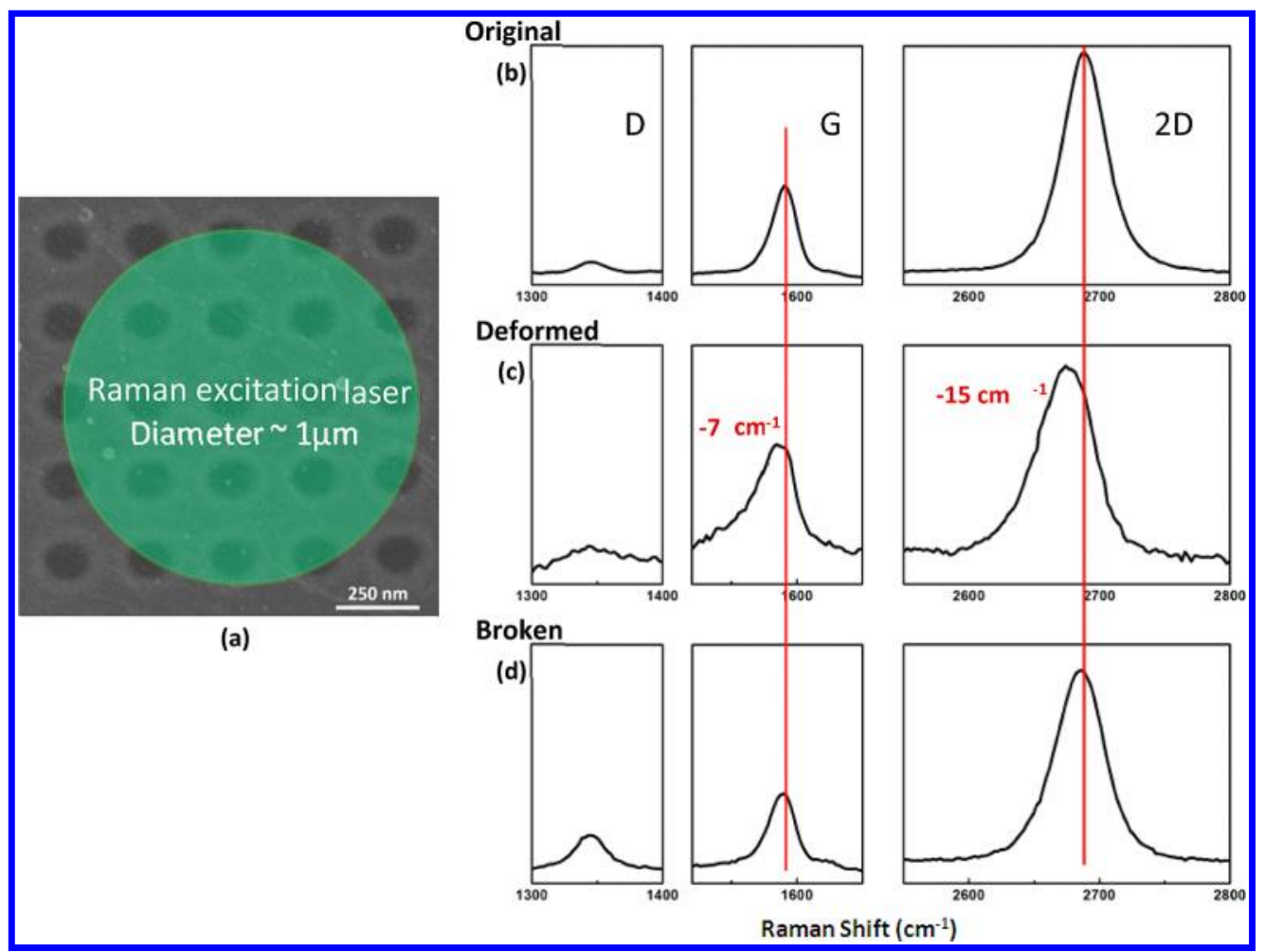

Figure 4. (a) Schematic of Raman excitation laser beam relative to graphene sample on circular molds. (b-d) Comparison of Raman D, G, and 2D peaks on unstrained region, max-strained region, and strained above limit region. The Raman shift is $15 \mathrm{~cm}-1$ after laser straining, as shown in (c) when the circular mold of $250 \mathrm{~nm}$ in diameter and $30 \mathrm{~nm}$ in depth is used. The Raman shift after breaking occurs is zero, as shown in (d) when the mold is $250 \mathrm{~nm}$ in diameter and $60 \mathrm{~nm}$ in depth.

place at the edge of the mold with aspect ratio is increased to 0.15 , and becomes more severe at a higher aspect ratio of 0.2 . The round piece was pulled off at aspect ratio of 0.3 . The experimental observation agrees well with $\mathrm{MD}$ simulation results of the graphene forming process, ${ }^{16}$ in which the graphene film first bulges by the pressure, and then fracture takes place at the edge and gradually becomes larger with the continuous increasing of straining. The deformed part of the film is pulled off at the edge. The reason that the fracture occurs at the edge of the mold is that the graphene film turns sharply while bending into the mold cavity, resulting in severe tension between the carbon atoms and high local strain.

The relationship between the mold diameter and critical aspect ratio is plotted in Figure 3c. Green circles are the highest safe aspect ratios the graphene film can be deformed to in experiment, and the area below the green circles is the safe forming area. The aspect ratios at which breaking of graphene were observed are marked by red crosses in the figure. The critical aspect ratio should be located between the red crosses and the green circles. The highest aspect ratio and corresponding strains reaches 0.25 and $12 \%$, respectively, for a $50 \mathrm{~nm}$ circular mold. The maximum aspect ratio the graphene gradually increases with the decreasing of the mold diameter. When the mold diameter is smaller than $100 \mathrm{~nm}$, the increasing rate of the aspect ratio becomes higher. The increasing of maximum aspect ratio with decreasing of mold size can be explained by the level of uniformity of the strain over the entire deformed area of graphene. The corresponding average strain of the safe aspect ratios (green circles) are plotted in the inlet of Figure 3a. It can be seen that with the decreasing of the mold size, the average strain in the deformed graphene before breaking increases. When the mold size is relatively large (over
$100 \mathrm{~nm}$ ), the carbon atoms at the edge experienced very high local strain while the others close to the center deforms little. Therefore, when the strain at the edge is high enough to cause fracture, the overall deformed aspect ratio/strain is still low. With the decreasing of mold size, the amount of carbon atoms in the forming area decreases and the portion of edge atoms are larger, so the strain across the whole area becomes more and more uniform, resulting in higher forming aspect ratio. It can be predicted that in an ideal and extreme case with only several carbon atoms being deformed, each $\mathrm{C}-\mathrm{C}$ bond can be elongated to the theoretical ultimate strain.

The study of the maximum aspect ratio is of important meaning as guidance for carrying out nanostrain engineering of graphene in the future. Besides the straining limit, the critical pressure that the graphene film can sustain is another important parameter to study. Circular molds with deep depth (deeper than the maximum aspect ratio that the graphene can attain) are used to study the critical pressure. Increasing laser intensity (shock pressure) is applied on the suspending graphene film and observed afterward, as seen in Figure 3b. It can be seen that when the pressure is increased to a low value, there is no obvious change in the film. As the hole is deep, although the pressure is enough to deform the graphene film, there is no surface for the film to attach to and hold its strained shape. Thus, after the release of the force the graphene would bounce back to the unstrained state. Once the exerted pressure is larger than the critical pressure, the graphene film will be broken. The critical pressure is related to the mold diameter in nanoscale, which was given in Figure 3c. It is found that the critical pressure increases slowly in the diameter range of 100 and 500 nm. In order to safely shape the graphene film into corresponding mold, smaller pressure should be used. The 


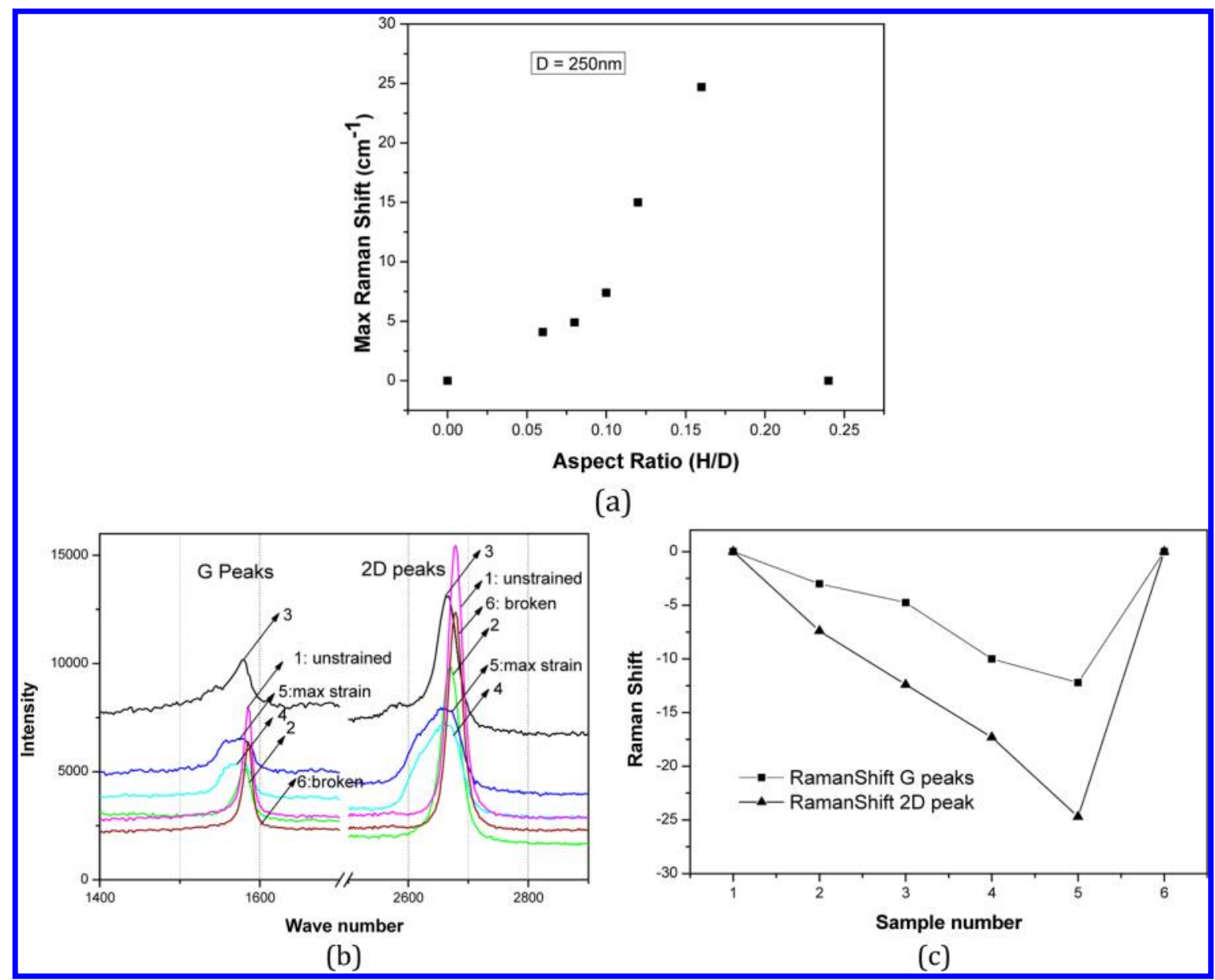

Figure 5. (a) The relationship between aspect ratio of mold $(D=250 \mathrm{~nm}, H$ varies from 0 to $60 \mathrm{~nm})$ and Max Raman shift. (b) Comparison of Raman G and 2D peaks on unstrained region (sample 1), several laser-strained region (for mold $250 \mathrm{~nm}$ in diameter and $40 \mathrm{~nm}$ in depth, several points (samples 2-5) were measured in the same sample with $\sim 1 \mu \mathrm{m}$ step size), and strained above limit regions (sample 6). The max straining occurs in sample 5 when the Raman shift is $24.7 \mathrm{~cm}^{-1}$. (c) The Raman shifts for various samples (numbers indexed in panel b).

breaking process of the graphene film is useful for nanopatterning application, and more details can be found in ref 16 .

The straining of the graphene film are further studied by Raman spectroscopy. The Raman spectra of unstrained graphene, graphene max-strained, and graphene strained above limit on circular mold with $250 \mathrm{~nm}$ diameter was measured, as plotted in Figure $4 \mathrm{~b}-\mathrm{d}$. The Raman excitation laser beam $(\lambda=532 \mathrm{~nm})$ is about $1 \mu \mathrm{m}$ and thus is larger than the mold diameter and covers an area with several molds, as illustrated by Figure $4 a$. It can be seen from Figure $4 b$ that the original CVD graphene sample is a typical single layer graphene $^{20-23}$ with the characteristic features as D, G, and $2 \mathrm{D}$ peaks, which lie at 1346,1590 , and $2689 \mathrm{~cm}^{-1}$, respectively. The $\mathrm{D}$ peak is very small, indicating a good quality of the film. The circular mold used is $250 \mathrm{~nm}$ in diameter and $30 \mathrm{~nm}$ in depth, corresponding to a forming aspect ratio of 0.12 and average strain of $3.8 \%$ of the strained area of graphene. After the graphene film is strained to aspect ratio $(0.12)$ in circular molds in diameter of $250 \mathrm{~nm}$, the $\mathrm{G}$ peak downshifts $7 \mathrm{~cm}^{-1}$ to $1583 \mathrm{~cm}^{-1}$, and the $2 \mathrm{D}$ peak down shifts $15 \mathrm{~cm}^{-1}$ to $2674 \mathrm{~cm}^{-1}$. The Raman frequency shifts verify the forming and strain induced in the graphene membrane. ${ }^{21-23}$ In addition, besides downshift, the $G$ peak of deformed graphene film also show slight broadening on the left part and becomes unsymmetrical. It is a sign of splitting of $G$ peak, which is also a strain effect. ${ }^{21}$ The Raman spectrum of fractured graphene film is plotted in
Figure $4 d$, in which the graphene film is deformed into $250 \mathrm{~nm}$ wide and $60 \mathrm{~nm}$ deep circular mold (aspect ratio $=0.24$ ). No obvious downshifts of $\mathrm{G}$ peak and $2 \mathrm{D}$ peak are observed. This is because after broken the strain induced in the deformed part of graphene is released. Meanwhile, an obvious increase of D peak is seen, indicating an increase of disorder and defect in the test area, and thus verifies the fracture of the graphene film.

The relationship between aspect ratio of mold $(D=250 \mathrm{~nm}$, $H$ varies from 0 to $60 \mathrm{~nm}$ ) and Max Raman shift (2D band) is plotted in Figure 5a, where the max Raman shift of $24.4 \mathrm{~cm}^{-1}$ occurs in aspect ratio of 0.16 . When the samples are strained above the limit, as shown in Figure 5a, aspect ratio of 0.24, and Figure $5 b$, sample 6, the Raman shift is zero. The Raman spectra of graphenes in unstrained region and several strained conditions were plotted in Figure 5b. In order to measure the variance of Raman shift as a function of position, several points were measured in the sample with $\sim 1 \mu \mathrm{m}$ step size $(D=250$ $\mathrm{nm}, H=40 \mathrm{~nm}$ ). The Raman shift of $2 \mathrm{D}$ peak varies from 7 to $24.7 \mathrm{~cm}^{-1}$ (as shown in Figure 5c). The variance of Raman shift is because the Raman excitation laser beam of $\sim 1 \mu \mathrm{m}$ is larger than the mold diameter and covers an area with several molds. In addition, compared to the average strain in graphene (3.8\%), the Raman shifts are relatively small, which corresponds to a strain level less than $0.8 \%$. There are two reasons: (1) the excitation beam covers a larger area than the strained area, and thus collects a sum signal both from unstrained and strained 
parts; and (2) the strain induced in the bent area is not uniform. When pushed into the circular molds, the graphene film is heavily strained at the edges of the trenches and shoulders near side walls, where the signal can hardly be collected. Thus, only signals from flat areas of graphene which are either slightly strained (bottom of shaped area) or unstrained (bar areas without mold cavities) are collected, resulting small shift in Raman spectrum.

As the laser beam size used is in millimeter scale, it allows for scalable treatment of the graphene surface while inducing nanoscale local straining in the graphene film. The positions, numbers, and amounts of local straining are controlled by the size and shapes of the nanopatterns on the substrate surface. Combining with the existing scalable patterning technique, such as photolithography, laser interference lithography, and E-beam lithography, the treated area in the proposed laser straining process could be further scaled up to roll to roll or plate to plate processes by adjusting the laser beam size and the scanning speed.

In summary, $3 \mathrm{D}$ shaping of CVD grown graphene film in nanoscale patterns using laser shock pressure was carried out, which induces local straining in the graphene film. The straining limit of the graphene film and the critical breaking pressure were studied. It is found that the graphene film can deform to an aspect ratio larger than 0.1 , and the critical breaking pressure is higher than $1 \mathrm{GPa}$. Both values were found to increase with the decreasing of mold size. The local straining and breaking of graphene film are verified by Raman spectra. Large scale pressing of the graphene film into nanoscale local patterns was presented. The process could be scaled up to roll to roll process by changing laser beam size and scanning speed. The proposed laser shock straining approach is fast, efficient, and easy, which has great potential to realize all graphene circuit for applications of graphene in nanoelectrical devices.

Methods. Setup of Laser Dynamic Forming. The laser shock process is similar to the one used in laser dynamic forming of thin films. The setup includes a laser source, a transparent confinement, a layer of ablative coating and an ultrathin metal foil, as illustrated in Figure 1a). A short pulsed Q-switch Nd:YAG (Continuum Surelite III) is used as the energy source. The laser beam has a Gaussian distribution and the pulse width is $10 \mathrm{~ns}$. A focus lens is used to control the bam size. The beam diameter used is $3 \mathrm{~mm}$, which is calibrated by a photosensitive paper (Kodak Linagraph, type: 1895). Glass slide was used as the confining media, and aerosol graphite painting (Asbury Carbons, U.S.A.) was sprayed on $4 \mu \mathrm{m}$ thick aluminum foil (Lebow Company Inc., Bellevue, WA) as the ablative coating. The thickness of the ablative coating is in the range from 1 to $10 \mu \mathrm{m}$. An $X-Y$ stage is used to move the processing stage.

Graphene Synthesis and Transfer. Our graphene samples are grown by $\mathrm{CVD}\left(\mathrm{CH}_{4}\right.$ as carbon stock) on $\mathrm{Cu}$ foils $(25 \mu \mathrm{m}$ thick, Alfa Aesar, 99.8\%) at ambient pressure. The $\mathrm{Cu}$ foil was loaded into a CVD furnace and heated to $1050{ }^{\circ} \mathrm{C}$ in $460 \mathrm{sccm}$ of flowing $5 \% \mathrm{H}_{2} / \mathrm{Ar}$. After $1050{ }^{\circ} \mathrm{C}$ was attained, the sample was annealed for at least $30 \mathrm{~min}$ without changing the gas flow rates. Then $\mathrm{CH}_{4}$ (concentration $500 \mathrm{ppm}$ ) was injected into the furnace at $50 \mathrm{sccm}$ for $90 \mathrm{~min}$ before the system was cooled to room temperature under protection of $\mathrm{Ar}$ and $\mathrm{H}_{2}$. After the growth process, the graphene samples were transferred by a PMMA-assisted process in a $\mathrm{Cu}$ etchant (iron nitrate) onto patterned Si mold. ${ }^{17}$
Preparation of Silicon Mold. Two methods are used prepare mold by cutting patterns on silicon wafers. The first one is focused ion beam (FIB) milling performed by FEI Nova 200 Nano-Lab DualBeam TM-SEM/FIB. The second method is electron beam lithography (EBL) using Leica VB-6 ultrahigh resolution, extra-wide field electron beam lithography tool. PMMA is used as the resist and is developed away after exposure. The rest of the resist is left on the surface of the silicon wafer, playing the role of cushion for shock absorbing in LDF.

Characterization Methods. Raman spectroscopy was performed using a HORIBA Jobin Yvon XploRA confocal Raman microscope equipped with a motorized sample stage from Märzhäuser Wetzlar (00-24-427-0000). The wavelength of the excitation laser was $532 \mathrm{~nm}$ and the power of the laser was kept below $1.3 \mathrm{~mW}$ without noticeable sample heating. The laser spot size was $\sim 1 \mu \mathrm{m}$ with a $100 \times$ objective lens. The SEM image was recorded on a Hitachi S-4800 field-emission scanning electron microscope or a FEI Nova 200 Nano-Lab DualBeam TM-SEM/FIB. The AFM images and height profile were measured by a DI Dimension 3100 atomic force microscope.

\section{AUTHOR INFORMATION}

\section{Corresponding Author}

*E-mail: gjcheng@purdue.edu.

\section{Notes}

The authors declare no competing financial interest.

\section{ACKNOWLEDGMENTS}

J.L. and G.J.C. want to thank supports from U.S. NSF (Grant CMMI 0928752) and CAREER Award (Grant CMMI-401 0547636) through program of Materials Processing and Manufacturing. T.F.C. and Y.P.C. acknowledges partial support by NSF DMR 0847638 and DTRA HDTRA1-09-1-0047.

\section{REFERENCES}

(1) Novoselov, K. S.; Geim, A. K.; Morozov, S. V.; Jiang, D.; Zhang, Y.; Dubonos, S. V.; Crigorieva, I. V.; Firsov, A. A. Electric field effect in atomically thin carbon films. Science 2004, 306, 666-669.

(2) Geim, A. K.; Novoselov, K. S. The rise of graphene. Nat. Mater. 2007, 6, 183-91.

(3) Heersche, H. B.; Jarillo-Herrero, P.; Oostinga, J. B.; Vandersypen, L. M. K.; Morpurgo, A. F. Bipolar supercurrent in graphene. Nature 2007, 446, 56-9.

(4) Novoselov, K. S.; Geim, A. K.; Morozov, S. V.; Jiang, D.; Katsnelson, M. I.; Grigorieva, I. V.; Dubonos, S. V.; Firsov, A. A. Twodimensional gas of massless Dirac fermions in graphene. Nature 2005, 438, 197-200.

(5) Gunlycke, D.; Lawler, H. M.; White, C. T. Room-temperature ballistic transport in narrow graphene strips. Phys. Rev. B 2007, 75, 5.

(6) Zhao, Q.; Nardelli, M. B.; Bernholc, J. Ultimate strength of carbon nanotubes: a theoretical study. Phys. Rev. B 2002, 65, 144105.

(7) Meric, I.; Han, M. Y.; Young, A. F.; Ozyilmaz, B.; Kim, P.; Shepard, K. L. Current saturation in zero-bandgap, topgated graphene field-effect transistors. Nat. Nanotechnol. 2008, 3, 654-9.

(8) Han, M. Y.; Ozyilmaz, B.; Zhang, Y. B.; Kim, P. Energy Band-gap Engineering of Graphene Nanoribbons. Phys. Rev. Lett. 2007, 98, 206805.

(9) Ponomarenko, L. A.; Schedin, F.; Katsnelson, M. I.; Yang, R.; Hill, E. W.; Novoselov, K. S.; Geim, A. K. Chaotic Dirac Billiard in Graphene Quantum Dots. Science 2008, 320, 356-358.

(10) Pereira, V. M.; Castro Neto, A. H. Strain engineering of graphene's electronic structure. Phys. Rev. Lett. 2009, 103, 046801. 
(11) Gui, G.; Li, J.; Zhong, J. Band structure engineering of graphene by strain: first-principles calculations. Phys. Rev. B 2008, 78, 075435.

(12) Guinea, F.; Katsnelson, M. I.; Geim, A. K. Energy gaps and a zero-field quantum hall effect in graphene by strain engineering. Nat. Phys. 2009, 6 (1), 30-33.

(13) Levy, N.; Burke, S. A.; Meaker, K. L.; Panlasigui, M.; Zettl, A.; Guinea, F.; Castro Neto, A. H.; Crommie, M. F. Strain-induced pseudo-magnetic fields greater than $300 \mathrm{~T}$ in graphene nanobubbles. Science 2010, 329, 544-547.

(14) Gao, H.; Ye, C.; Cheng, G. J. Forming behaviors and critical parameters of microscale laser dynamic forming. Trans. ASME J. Manu. Sci. Eng. 2009, 131, 051011.

(15) Li, J.; Gao, H.; Cheng, G. J. Forming Limit and Fracture Mode of Microscale Laser Dynamic Forming. Trans. ASME J. Manu. Sci. Eng. 2010, 108, 013107.

(16) Li, J.; Zhang, R.; Jiang, H.; Cheng, G. J. Scalable nano-patterning of graphenes using laser shock. Nanotechnology 2011, 22, 475303.

(17) Cao, H. L.; Yu, Q. K.; Jauregui, L. A.; Tian, J. F.; Wu, W.; Liu, Z. H.; Jalilian, R.; Benjamin, D. K.; Jiang, Z.; Bao, J. M.; Pei, S. S.; Chen, Y. P. Electronic Transport in Chemical Vapor Deposited Graphene Synthesized on $\mathrm{Cu}$ : Quantum Hall Effect and Weak Localization. Appl. Phys. Lett. 2010, 96, 122106.

(18) Lee, C.; Wei, X. D.; Kysar, J. W.; Hone, J. Measurement of the elastic properties and intrinsic strength of monolayer graphene. Science 2008, 321, 385-8.

(19) Fabbro, R.; Fournier, J.; Ballard, P.; Devaux, D.; Virmont, J. Physical study of laser-produced plasma in confined geometry. J. Appl. Phys. 1990, 68, 775-84.

(20) Ferrari, A. C.; Meyer, V. S.; Casiraghi, C.; Lazzeri, M.; Mauri, F.; Piscanec, S.; Jiang, D.; Novoselov, K. S.; Roth, S.; Geim, A. K. Raman spectrum of graphene and graphene layers. Phys. Rev. Lett. 2006, 187401.

(21) Mohiuddin, T. M. G.; Lombardo, A.; Nair, R. R.; Bonetti, A.; Savini, G.; Jalil, R.; Bonini, N.; Basko, D. M.; Galiotis, C.; Marzari, N.; Novoselov, K. S.; Geim, A. K.; Ferrari, A. C. Uniaxial strain in graphene by Raman spectroscopy: G peak splitting, Gruneisen parameters, and sample orientation. Phys. Rev. B 2009, 79, 205433.

(22) Ding, F.; Ji, H.; Cheng, Y.; Herklotz, A.; Dorr, K.; Mei, Y.; Rastelli, A.; Schmidt, G. Stretchable graphene: a close look at fundamental parameters through biaxial straining. Nano Lett. 2010, 10, 3453-3458.

(23) Zabel, J.; Nair, R. R.; Ott, A.; Georgiou, T.; Geim, A. K.; Novoselov, K. S.; Casiraghi, C. Raman spectroscopy of graphene and bilayer under biaxial strain: bubbles and balloons. Nano Lett. 2012, 12, $617-621$. 